\title{
Case 2 - Advanced low-grade ovarian cancer with peritoneal implants
}

\author{
Annalisa Garbi ${ }^{1}$, Maria Teresa Achilarre ${ }^{1}$, Maria Cristina Petrella ${ }^{1}$
}

\begin{abstract}
Low-grade serous carcinomas are a less common subtype of serous epithelial ovarian cancer. They differ from high-grade serous tumors in their immuno-histochemical profile, epidemiologic features, clinical behavior and molecular characteristics. This case report describes the clinical history of a young woman who underwent multiple surgical and medical treatments.
\end{abstract}

Key words: chemoresistance, cytoreductive surgery, invasive low-grade serous ovarian cancer, MEK inhibitors

\section{Introduction}

Serous carcinoma is the most common histologic subtype of ovarian cancers. A 2-tier system of grading was proposed to separate them into low-grade serous (LGS) or high-grade serous (HGS) ovarian carcinoma $[1,2]$. LGS carcinomas are far less common (10\%) than HGS carcinomas and they differ in their immuno-histochemical profile, epidemiologic features, clinical behavior and molecular characteristics. Patients with LGS ovarian carcinoma are generally characterized by young age at diagnosis, relative chemoresistance, and prolonged overall survival (OS) [3]. LGS can arise either de novo, most frequently diagnosed at an advanced stage, or following a diagnosis of serous borderline tumor. Despite these substantial differences, treatment of LGS cancer is similar to that used for HGS epithelial ovarian cancer, and it involves a combination of cytoreductive surgery followed by platinum based chemotherapy.

At the time of relapse, patients can be treated with surgery, chemotherapy and/or hormonal therapy. There is increasing evidence of a distinct molecular genetic pathway for LGS carcinoma; in particular, the mitogen-activated protein kinase pathway appears to have a major

'Division of Gynecologic Oncology, European Institute

of Oncology, Milan, Italy.

Correspondence to: Garbi Annalisa, MD

Division of Gynecologic Oncology, European Institute of Oncology, Via Ripamonti 435, 20141 Milano, Italy.

Phone: +39 0257489543 - Fax: +39 0294379222

E-mail: annalisa.garbi@ieo.it

CANCER BREAKING NEWS 2016;4(2):48-53

DOI: $10.19156 / \mathrm{cbn} .2016 .0018$ role in its pathogenesis. In contrast to HGS, which is characterized by a significantly higher frequency of protein p53 mutation, a number of genetic alterations have in fact been found to be associated with LGS ovarian carcinogenesis: specifically, KRAS and BRAF mutations occur with a frequency of $35 \%$ and $33 \%$, respectively [4]. Due to the frequency of $R A S$ or $B R A F$ mutations, the use of a MEK inhibitor, thereby interfering with the RAS/ $\mathrm{RAF} / \mathrm{MEK} / \mathrm{ERK}$ pathway, is under evaluation as an alternative to standard chemotherapy in recurrent LGS. Furthermore, mutational status appears to be a potential prognostic factor in low-grade serous carcinoma of the ovary or peritoneum [3].

\section{Case report}

This case report describes a 27 year-old woman with no relevant comorbidities, nulliparous and with a strong desire to conceive. Her oncological family history was negative. Due to abnormal uterine bleeding in 2007 she underwent transvaginal ultrasound scan showing a $7 \mathrm{~cm}$ uniloculatesolid cyst on the left ovary with vascularized papilla (color score $=3$ ) and crescent sign (residual ovarian parenchyma), suspicious for borderline tumor. She underwent conservative surgical treatment by laparoscopic removal of the cyst and comprehensive endoperitoneal staging procedures. Final pathology confirmed the diagnosis of serous borderline tumor with micropapillary pattern and foci of low-grade non-invasive adenocarcinoma. Desmoplastic peritoneal implants were also described. Final International Federation of Gynecology and Obstetrics (FIGO) stage was IC due to iatrogenic spillage of the cyst content during surgery. The patient did not receive any adjuvant treatment. 

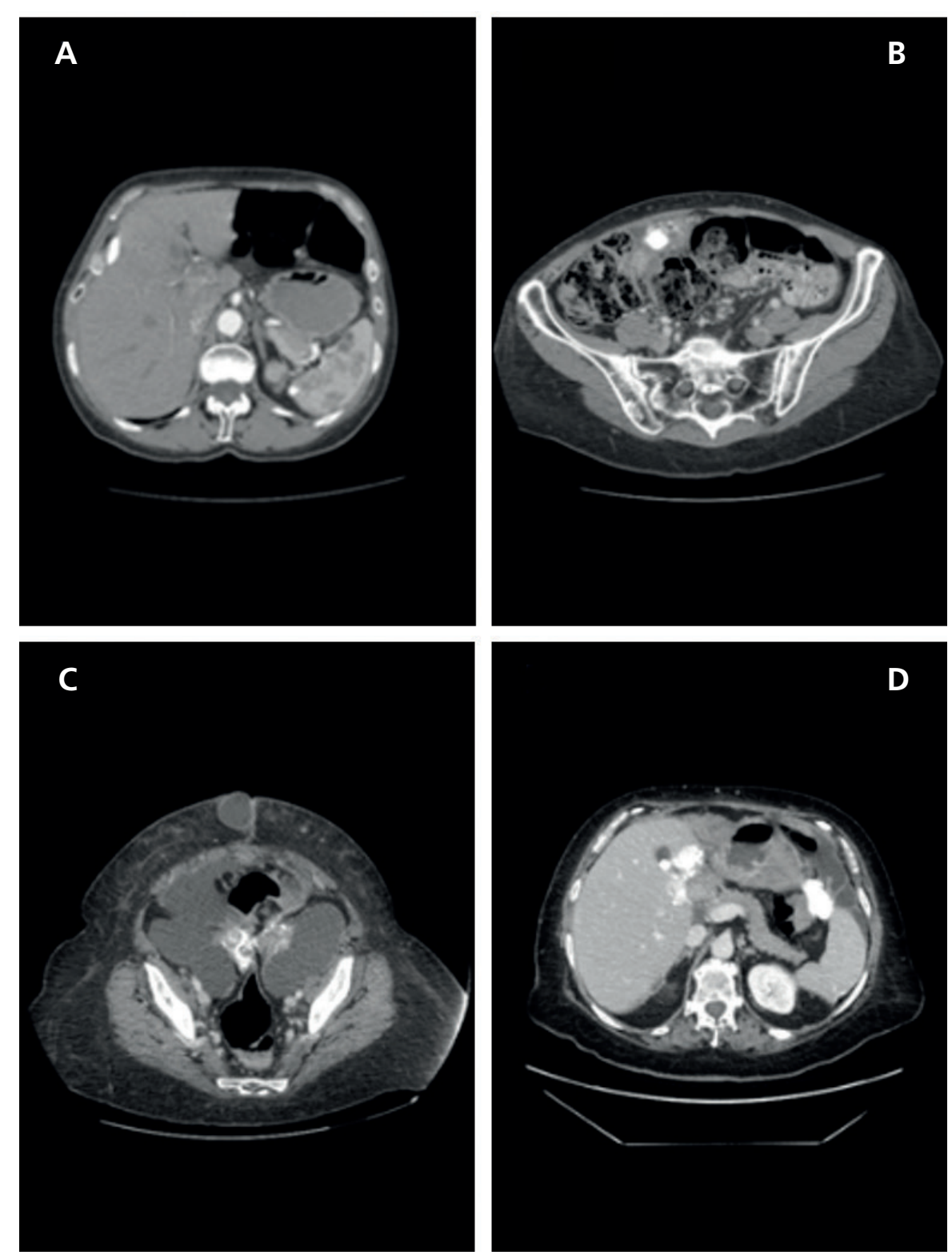

Fig. 1. Preoperative computed tomography (CT) scan showing (A) calcific implants (psammoma bodies) next to the Glisson's capsule, (B) omental implant in the hypogastrium, (C) bilateral pelvic mass with calcific components, and (D) peritoneal implants next to the gastrosplenic ligament, Glisson's capsule and falciform ligament.

Twenty months later (in 2009) she presented with evidence of relapse on the contralateral ovary showing the same ultrasound findings described at the first diagnosis and, therefore, she again underwent conservative minimally invasive surgery showing the same histopathological patterns. She then received adjuvant hormonal treatment with tamoxifen and gonadotropin-releasing hormone analogs for one year. During the subsequent follow-up, a significant increase of cancer antigen 125 (CA125) was observed and for this reason in December 2011 she underwent the third laparoscopic surgery with findings of diffuse peritoneal implants and bilateral adnexal involvement. Final pathology results on peritoneal, adnexal and endometrial biopsies revealed invasive LGS adenocarcinoma. A second opinion was obtained from a world recognized expert in gynecologic pathol- ogy (J Pratt), who highlighted "the progression from the serous borderline ovarian tumor to low-grade serous tumor (with invasive implants). Unfortunately this tumor has low chemo-sensitivity due to the absence of mitosis and slow proliferation rate, therefore the best option remains surgery".

In January 2012 she was referred to our Institute for a third opinion. After multidisciplinary discussion and exhaustive counselling with the patient a demolitive cytoreductive surgery was planned. A preoperative computed tomography (CT) scan demonstrated diffuse abdominal and pelvic peritoneal disease with bilateral adnexal masses, both showing the typical pattern with calcific implants (Figure 1).

The patient underwent extensive cytoreductive surgery in the lower and upper abdomen with radical oophorectomy, hysterectomy, multiple bowel resections, stripping 

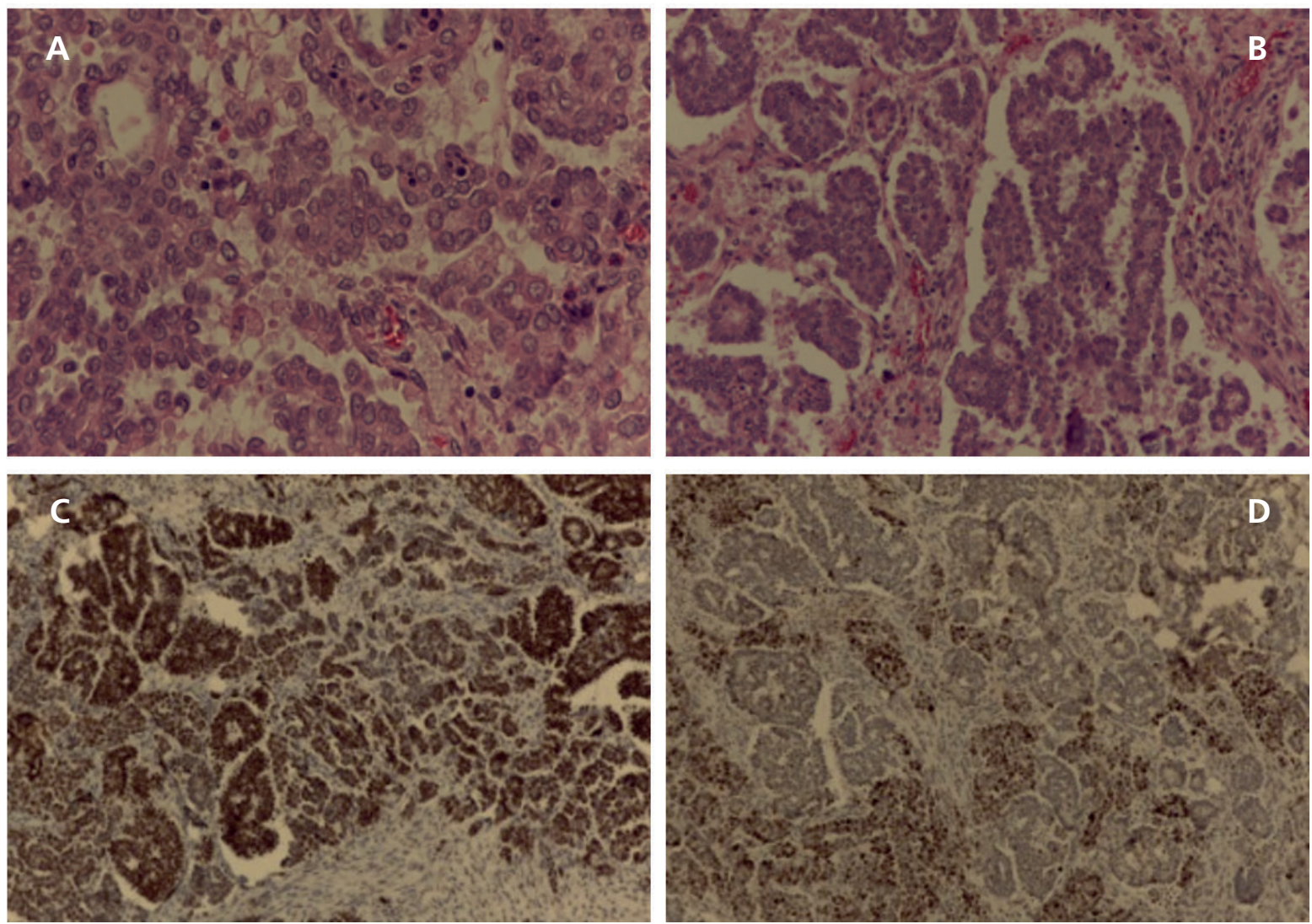

Fig. 2. Histopathological features: (A, B) abundant psammoma bodies and low mitotic activity, (C) strong and diffuse nuclear expression of Wilms' tumor suppressor gene (WT1), (D) absence of TP53 mutation.

and resection of the right diaphragm, and port site excision, with no macroscopic residual tumor left at the end of the procedure. Histopathological findings confirmed an advanced low-grade ovarian cancer. Macroscopically, the case demonstrated a bilateral ovarian involvement with a fine papillary growth. Microscopically, the neoplasm was characterized by micropapillae haphazardly infiltrating the stroma and surrounded by a characteristic unlined clear space. Macropapillae, sheets, small glands, and cysts were also observed. The cells showed mild to moderate nuclear atypia ( $<3$-fold variation in nuclear size) with limited nuclear pleomorphism and rare prominent nucleoli. Abundant psammoma bodies were present. Mitotic activity was low ( $<2-3 \mathrm{mf} /$ per $10 \mathrm{hpf}$ ) (Figure 2A, B). The neoplastic population had a strong and diffuse nuclear expression of Wilms' tumor suppressor gene (WT1), as observed in both low- and high-grade serous tumors (Figure 2C). On the contrary, the nuclear staining pattern of $\mathrm{p} 53$ was of the "wild-type", with only a focal and irregular positivity. This type of expression correlates with the absence of the TP53 mutation which, instead, is present in virtually all cases of high-grade serous tumors (Figure 2D).
A molecular analysis for $K R A S$ (codon 12, 13 e 61), PI3K (esone 9 e 20), BRAF (esone 11 e 15) and HER-2/neu genes was negative.

According to international guidelines (National Comprehensive Cancer Network [NCCN] and European Society for Medical Oncology [ESMO]) she received six cycles of three-weekly adjuvant chemotherapy based on carboplatin and paclitaxel, which was completed in July 2012 with no evidence of disease at the end of treatment.

Follow-up involving regular clinical, biochemical and instrumental evaluations was carried out for the following two years when, unfortunately, a CT scan showed a millimetric calcific implants on the Glisson's capsule (glissoniana), a $27 \mathrm{~mm}$ lesion in the right paracolic gutter and a symptomatic $10 \mathrm{~cm}$ mass in the pelvis.

Considering the patient's clinical history, the treatments already received and the platinum-sensitive relapse, a rechallenge with platinum (carboplatin and pegylated liposomal doxorubicin) was administered. A partial response was observed after three courses, but the CT scan performed after completing the six cycles highlighted stable disease. A multidisciplinary discussion recommended 
a further surgery, therefore in August 2014 the patient underwent a secondary cytoreduction with no evidence of disease at the end of surgery. At this time hormonal treatment with anastrozole ( $1 \mathrm{mg} /$ day $)$ was considered as maintenance therapy until January 2015 when a new pelvic relapse was diagnosed.

Given the chemotherapy resistance and the number of previous surgical treatments the patient was evaluated to be enrolled in clinical trials with MEK inhibitors, despite the absence of KRAS/BRAF mutation. She was randomized to receive MEK162 (45 mg orally twice daily) but after two months she underwent disease progression, therefore weekly paclitaxel was recommended. She received six cycles of chemotherapy until September 2015 with stable disease but the treatment was interrupted due to severe neuropathy. The patient was started on treatment with tamoxifen that is still ongoing; clinical conditions are stable, and a new clinical evaluation is scheduled in the near future.

\section{Conclusion}

This case highlights the indolence of invasive LGS ovarian cancer. Recurrence after a primary diagnosis of serous tumor of low malignant potential such as LGS is frequent as in our case and it ranges from $75 \%$ to $80 \%$ of cases $[5,6]$. KRAS and BRAF mutations occurred with a fre-

\section{References}

1. Malpica A, Deavers MT, Lu K et al. Grading ovarian serous carcinoma using a two-tier system. Am J Surg Pathol 2004;28:496-504.

2. Bodurka DC, Deavers MT, Tian C et al. Reclassification of serous ovarian carcinoma by a 2-tier system: a Gynecologic Oncology Group Study. Cancer 2012;118:3087-94.

3. Gershenson DM, Bodurka D, Lu K et al. The impact of age and primary disease site on outcome of women with lowgrade serous carcinoma of the ovary or peritoneum: Results of a large single-institution registry of a rare tumor. J Clin Oncol 2015;33:2675-82.

4. Farley J, Brady WE, Vathipadiekal V et al. Selumetinib in women with recurrent low-grade serous carcinoma of the quency of $35 \%$ and $33 \%$, respectively, however more recent reports indicated a much lower frequency of these molecular alterations. As well as for HGS tumors, surgery remains the cornerstone in the treatment of LGS counterparts, and performing a maximal cytoreductive effort is paramount for women with this disease. According to data reported by Crane in 2015, secondary surgery with maximally cytoreductive effort is also to be considered [7]. Furthermore, our clinical case confirms a more favorable overall prognosis for LGS tumors compared to the HGS ovarian cancer population, and a well-known resistance to conventional medical treatments. MEK inhibitors are in the process of being evaluated as possible alternative agents. However, ongoing studies have not yielded the expected results in term of progression-free survival, therefore different strategies are still needed.

\section{Acknowledgments}

The authors thank Ray Hill, an independent medical writer, who provided native English editing and journal styling on behalf of HPS. This editorial assistance was funded by PharmaMar, Spain.

\section{Conflicts of Interest}

The Authors declare there are no conflicts of interest in relation to this article. ovary or peritoneum: an open-label, single-arm, phase 2 study. Lancet Oncol 2013;14:134-40.

5. Crispens MA, Bodurka D, Deavers M et al. Response and survival in patients with progressive or recurrent serous ovarian tumors of low malignant potential. Obstet Gynecol 2002;99:3Y10.

6. Silva EG, Gershenson DM, Malpica A et al. The recurrence and the overall survival rates of ovarian serous borderline neoplasms with noninvasive implants is time dependent. Am J Surg Pathol 2006;30:1367-71.

7. Crane EK, Sun CC, Ramirez PT et al. The role of secondary cytoreduction in low-grade serous ovarian cancer or peritoneal cancer. Gynecol Oncol 2015 Jan;136(1):25-9. 


\section{Commentary}

Low-grade serous ovarian cancer (LGSOC) is a rare disease which represents only 5-8\% of all ovarian cancers and 6-10\% of all serous ovarian carcinomas [1-3]. Since the introduction of the two tier system by Malpica et al., it has become clear that LGSOC has distinct clinical, histological and molecular characteristics, compared with the high-grade counterpart [4, 5]. LGSOC merits a different clinical approach. The above article stresses the difference between LGSOC and high-grade serous ovarian cancer (HGSOC) and the importance to deviate from classical epithelial ovarian cancer treatment towards a more tailored one.

The young age at onset in this case are typical for LGSOC, with a median age of 43-55 years at diagnosis compared to 63 years in HGSOC [6, 7]. The patient was initially diagnosed with a serous borderline tumor of the micropapillary type with foci of low-grade non-invasive adenocarcinoma. Recurrences of borderline tumors as invasive carcinomas are rare, about 5\%, but the micropapillary type has been described as one of the possible risk factors for invasive recurrence [8].

LGSOC harbours a relative chemo-resistance, making surgery a key cornerstone in the treatment, in first line as well as in relapse. In 2008, Schmeler et al. published their response rates of neo-adjuvant chemotherapy in 25 cases of primary inoperable LGSOC cases. Response rates were poor, with only one patient achieving complete remission, none with partial remission and 21 with stable disease [9]. Nevertheless, the use of neo-adjuvant and adjuvant chemotherapy has not yet been abandoned in first line. Surgery should always be considered in LGSOC recurrence with progression-free survival (PFS) $>6$ months, and neo-adjuvant chemotherapy in LGSOC should be used in a restricted manner. Tumorigenesis in LGSOC is RAS-signaling related, characterized by KRAS and BRAF mutations. The published mutation rates of KRAS and $\mathrm{BRAF}$ differ between different publications with a large range: 19-54\% for KRAS mutations and 2-33\% for BRAF mutations [10-12]. It is suggested that a BRAF mutation is associated with early stages of the disease, hence explaining the broad mutation rate. In light of these findings and the relative chemo-resistance, clinical trials with therapies targeting the RAS-pathway were conducted. Farley et al. published the first phase II study of the MEKinhibitor selumetinib in recurrent LGSOC [13]. Overall response rate was $15 \%$ with a median PFS of 11 months. Two other trials with MEK-inhibitors in LGSOC (NCT 01849874 and NCT 02101788) are currently ongoing. The authors included this patient in the MEKI-trial even with no proven mutation. Mutational status has not been proven predictive for response of MEK-inhibitors [13], so inclusion was certainly justified.

The patient in this case received two hormonal treatments thus far. Gershenson et al. published the largest retrospective series of 64 LGSOC patients treated with different hormonal regimens, resulting in a combined overall response rate of $9 \%$, similar to response rates of chemotherapy, but with lower toxicity [14]. In addition, Gershenson et al. reported during the ASCO 2016 meeting on the improved PFS of patients treated with hormonal therapy after first-line chemotherapy [15].

LGSOC is a subtype of epithelial ovarian cancer with a good overall survival, but with a relative chemo-resistance making treatment of relapse more challenging. MEK-inhibitors and hormonal regimens are emerging as active agents, but further researches into robust predictive biomarkers are warranted. The combination of MEK- and PI3K-inhibitors should be further explored.

Els Van Nieuwenhuysen, Ignace Vergote

Division of Gynecologic Oncology

Department of Gynaecology and Obstetrics Leuven Cancer Institute, University Hospital of Leuven, Belgium E-mail: ignace.vergote@uzleuven.be 


\section{References}

1. Schmeler KM, Gershenson DM. Low-grade serous ovarian cancer: a unique disease. Curr Oncol Rep 2008;10(6):519-23.

2. Plaxe SC. Epidemiology of low-grade serous ovarian cancer. Am J Obstet Gynecol 2008;198(4):459.e1-8.

3. Gourley C, Farley J, Provencher DM et al. Gynecologic Cancer InterGroup (GCIG) consensus review for ovarian and primary peritoneal low-grade serous carcinomas. Int J Gynecol Cancer 2014;24(9 Suppl 3):S9-13.

4. Bodurka DC, Deavers MT, Tian C et al. Reclassification of serous ovarian carcinoma by a 2-tier system: a Gynecologic Oncology Group Study. Cancer 2012;118(12):3087-94.

5. Malpica A, Deavers MT, Lu K et al. Grading ovarian serous carcinoma using a two-tier system. Am J Surg Pathol 2004;28(4):496-504.

6. Gershenson DM, Sun CC, Lu KH et al. Clinical behavior of stage II-IV low-grade serous carcinoma of the ovary. Obstet Gynecol 2006;108(2):361-8.

7. Oswald AJ, Gourley C. Low-grade epithelial ovarian cancer: a number of distinct clinical entities? Curr Opin Oncol 2015;27(5):412-9.

8. Uzan C, Nikpayam M, Ribassin-Maied L et al. Influence of histological subtypes on the risk of an invasive recurrence in a large series of stage I borderline ovarian tumor including 191 conservative treatments. Ann Oncol 2014;25(7):1312-9.
9. Schmeler KM, Sun CC, Bodurka MT et al. Neo-adjuvant chemotherapy for low-grade serous carcinoma of the ovary or peritoneum. Gynecol Oncol 2008;108(3):510-14.

10. Wong KK, Tsang YT, Deavers MT et al. BRAF mutation is rare in advanced-stage low-grade ovarian serous carcinomas. Am J Pathol 2010;177(4):1611-7.

11. Singer G, Kurman RJ, Chang HW et al. Diverse tumorigenic pathways in ovarian serous carcinoma. Am J Pathol 2002;160(4):1223-8.

12. Singer G, Oldt R, 3rd, Cohen Y et al. Mutations in BRAF and KRAS characterize the development of low-grade ovarian serous carcinoma. J Natl Cancer Inst 2003; 95(6):484-6.

13. Farley J, Brady WE, Vathipadiekal V et al. Selumetinib in women with recurrent low-grade serous carcinoma of the ovary or peritoneum: an open-label, single-arm, phase 2 study. Lancet Oncol 2013;14(2):134-40.

14. Gershenson DM, Sun CC, Iyer RB et al. Hormonal therapy for recurrent low-grade serous carcinoma of the ovary or peritoneum. Gynecol Oncol 2012;125(3):661-6.

15. Gershenson DM, Bodurka DC, Coleman RL et al. Hormonal maintenance therapy for women with low grade serous carcinoma of the ovary or peritoneum [Abstract]. J Clin Oncol 2016;34(15 suppl):290 (Abstract 5502). 